\title{
Análise da Pele de Três Espécies de Peixes: Histologia, Morfometria e Testes de Resistência
}

\author{
Maria Luiza R. Souza1, Doroty M. Dourado², Silmara D. Machado ${ }^{3}$, Daniele F. Buccini ${ }^{3}$, Maria \\ Inês A. Jardim ${ }^{4}$, Rosemary Matias ${ }^{2}$, Celso Correia ${ }^{5}$, Isabel C. Ferreira ${ }^{6}$
}

\begin{abstract}
RESUMO - O objetivo deste trabalho foi analisar a pele de três espécies de peixes (piavuçu Leporinus macrocephalus, pacu prata Mylossoma sp e piraputanga Brycon hilarii) através de análise histologica e morfometrica e testes de resistência após o curtimento. Amostras de peles da região dorsal foram coletadas, fixadas em Bouin por 24 horas, incluídas em parafina, cortadas em $5 \mu \mathrm{m}$ de espessura e coradas por Hematoxilina-eosina. Os cortes foram analisados pela microscopia de luz. Foi utilizado o dinamômetro EMIC para a análise físico-mecânica do couro em 10 amostras no sentido longitudinal a partir da região dorsal do peixe de cada espécie. A derme desses peixes possui um padrão estrutural comum aos teleósteos, porém a disposição e distribuição das fibras colágenas apresentaram-se diferentes entre as espécies analisadas. Através da análise morfométrica constatou-se que a espessura da derme da pele diferiu entre as três espécies. Não houve diferença no teste de resistência à tração no couro da piraputanga $\left(16,88 \mathrm{~N} / \mathrm{mm}^{2}\right)$ e piavuçu $\left(18,50 \mathrm{~N} / \mathrm{mm}^{2}\right)$. Os resultados para os couros destas duas espécies de peixes foram superiores ao pacu $\left(11,83 \mathrm{~N} / \mathrm{mm}^{2}\right)$. O valor de alongamento até a ruptura foi superior para piavuçu $(52,83 \%)$ e piraputanga $(60,45 \%)$, não diferindo entre eles, porém foi inferior para o pacu $(33,83 \%)$. O rasgamento progressivo foi maior no couro de pacu $(36,51 \mathrm{~N} / \mathrm{mm})$. As peles das três espécies analisadas podem ser transformadas em couro para sua aplicação em diversos artefatos.
\end{abstract}

Palavras-chave: derme, resistência, fibras colágenas, histologia, morfometria, testes físico-mecânicos

\section{Analysis of the Skin of Three Fish Species: Histology, Morphometry and Resistance Tests}

\begin{abstract}
This study was carried out to analyse the skin of three species of fish (piavuçu Leporinus macrocephalus, pacu prata Mylossoma sp e piraputanga Brycon hilarii) through histological and morphometrical analysis and resistance tests after hardening. Skin samples from the dorsal region were taken and fixed in Bouin solution for 24 hours. They were embedded in paraffin, cut $5 \mu \mathrm{m}$ thick and stained by Hematoxylin-eosin technique. The cuts were analyzed by light microscopy. A EMIC dynamometer was used for the physical mechanical analysis of the skin in 10 samples, longitudinally oriented from the dorsal region of each specie of fish. The dermis of those fishes has a structural pattern common to teleosts, but the setting and distribution of collagen fibers was different among the analyzed species. The skin dermis thickness differed among the three species by the morphometrical analysis. No difference was observed for the resistance test to traction in piraputanga leather $\left(16.88 \mathrm{~N} / \mathrm{mm}^{2}\right)$ as well as in piavuçu leather $\left(18.50 \mathrm{~N} / \mathrm{mm}^{2}\right)$. The results for the skin of both fish species were higher than those for pacu $\left(11.83 \mathrm{~N} / \mathrm{mm}^{2}\right)$. The value of stretching as to the point of rupture was higher for piavuçu (52.83\%) and piraputanga (60.45\%), showing no difference among them, but it was lower for pacu $(33.83 \%)$. The progressive tearing was greater in pacu skin $(36.51 \mathrm{~N} / \mathrm{mm})$. The skin of the three analyzed species can be turned into leather for the manufacture of several artifacts.
\end{abstract}

Key Words: dermis, resistance, collagen fibers, histology, morphometry, physical mechanical tests

\section{Introdução}

A pele de peixe é considerada um subproduto, tornando-se um problema para o produtor ou para o abatedouro, pois, de acordo com Contreras-Guzmán (1994), a pele perfaz, em média, 7,5\% do peso dos peixes teleósteos. Muitas vezes, essas peles são moídas juntamente com vísceras e restos de carcaça do próprio animal e fornecida como fonte alimentar para animais.

A elaboração do couro a partir das peles residuais da filetagem de pescados representa uma fonte alternativa de renda que pode servir de matéria-

\footnotetext{
${ }^{1}$ Profa ${ }^{\text {. Dra }}$ do Depto de Zootecnia - Universidade Estadual de Maringá. Av. Colombo 5790, CEP: 87020-900, Maringá - PR, Brasil. E.mail:mlrsouza@uem.br

${ }^{2}$ Profa ${ }^{2}$ M.Sc. do Centro de Ciências Biológicas, Agrárias e da Saúde da Universidade para o desenvolvimento do Estado e da Região do Pantanal (UNIDERP).

${ }^{3}$ Acadêmica do Centro de Ciências Biológicas, Agrárias e da Saúde da UNIDERP

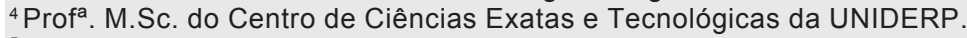

${ }^{5}$ Profa. Dr do Centro de Ciências Exatas e Tecnológicas da UNIDERP.

${ }^{6}$ Tecnóloga em Produção Química de Couros da empresa BASF S.A.
} 
prima para a fabricação de carteiras, biquinis, entre outros artefatos.

Das 100 mil toneladas de pescado beneficiado por ano para exportação ou consumo interno na Amazônia, 7 mil são peles e, conhecendo esse fato, o engenheiro de pesca Nilson de Aguiar, coordenador do projeto INPA decidiu buscar essa aplicação nobre para as sobras, aumentando o valor desse recurso biológico existente em grande quantidade nessa região (Adeodato, 1995).

Na confecção, as peles são classificadas por tamanho, para garantir melhor aproveitamento do couro, e cortadas para a fabricação das mantas, que atualmente são comercializadas por $\mathrm{m}^{2}$. Os couros de tilápias são comercializados por unidade, variando de 0,80 a 1,80 centavo de real ou na forma de mantas, cujo valor varia de $\mathrm{R} \$ 200,00$ a $\mathrm{R} \$ 250,00$ para mantas confeccionadas com couros semi-acabados.

A pele de peixe é um produto nobre e de alta qualidade, possuindo resistência como característica peculiar. Além dessa característica, para as espécies de peixes com escamas, as lamélulas de proteção, na inserção da escama (Almeida, 1998), resultam, após o curtimento, em um couro de aspecto típico e difícil de ser imitado, garantindo exclusiva padronagem de alto impacto visual (Adeodato, 1995).

O desenho exótico apresentado nas peles após curtimento compensa o seu reduzido tamanho. O desenho original dessas peles que dificilmente pode ser imitado por chapas de impressão sobre outros couros impede a falsificação desse tipo de produto, principalmente se as lamélulas de proteção e inserção das escamas forem mais alongadas.

No curtimento, é mantida a natureza fibrosa da pele, porém as fibras são previamente separadas pela remoção do tecido interfibrilar e pela ação de produtos químicos. Após a separação das fibras e remoção do material interfibrilar, as peles são tratadas com substâncias denominadas curtentes, que as transformam em couros ou peles processadas (Hoinacki, 1989).

Portanto, a pele é submetida a determinados processos, com utilização de produtos químicos e/ou vegetais, preservando-a da putrefação, ocasionado por processos autolíticos da própria pele ou ataque bacteriano. Assim, a pele é transformada em um material imputrescível, com características de maciez, elasticidade, flexibilidade, resistência à tração, enfim com determinadas qualidades físico-mecânicas, sendo denominado este processo de curtimento (Abracoa, 1995).

A diferença entre a pele e o couro pode ser definida como a pele sendo o tegumento que reveste o indivíduo antes do curtimento, enquanto o couro é a matéria-prima obtida após o curtimento, ou seja, após a adição do agente curtente na pele (Hoinacki, 1989). As peles de peixe diferem das peles de mamíferos, por estarem cobertas por delgadas epidermes, podendo ou não possuir escamas e não apresentam glândulas sebáceas (Junqueira, 1983b).

A derme, que é uma camada localizada abaixo da epiderme, é constituída por grossos feixes colágenos, dispostos paralelamente à superfície da pele e que estão entrelaçados de espaço em espaço por grossos feixes perpendiculares à superfície, em muitas espécies de peixes.

Existem diferenças acentuadas entre as peles das várias espécies de peixes, as quais necessitam de técnicas diferenciadas de curtimento, seja em tempo ou quantidade de produtos, acréscimo de um ou outro produto químico, até mesmo acréscimo de etapas (Adeodato, 1995). O colágeno é a proteína mais importante sob o ponto de vista do curtidor, pois reage com agentes curtentes (Hoinacki, 1989).

Estudos da resistência mecânica realizada no laboratório do IPT (Franca), BASF (RS) comprovou que os couros de peixe, cortados na mesma espessura do couro bovino, apresentam maior resistência (Adeodato, 1995; Sacco, 1998), tornando-se indispensável que se pense no aproveitamento integral dos subprodutos gerados pelo abate dos peixes, exemplo a pele.

O objetivo deste trabalho foi analisar a pele de três espécies de peixes presentes na região do Estado do Mato Grosso do Sul, por intermédio da histologia da pele e testes de resistência físicamecânica após o curtimento.

\section{Material e Métodos}

As espécies de peixes utilizadas foram piavuçu (Leporinus macrocephalus), piraputanga (Brycon hilarii) e pacu prata (Mylossoma sp.) obtidas no Projeto Pacu1, piscicultura situada em Terenos no Estado de Mato Grosso do Sul. De cada espécie foram utilizados 32 peixes pesando entre 400 e $800 \mathrm{~g}$.

\section{Captura dos peixes}

Os peixes foram coletados por meio de tarrafa com malha $\mathrm{n}^{\mathrm{o}} 12$ e colocados em gelo por 30 minutos, em seguida realizou-se a destruição da medula espinhal. Foi determinado o comprimento total (cm) utilizando-se um ictiômetro e pesados (g) em balanças com sensibilidade de $1 \mathrm{~g}$. 


\section{Análise histológica}

As amostras de pele para análise histológica foram retiradas da região dorsal média (entre a linha lateral e a nadadeira dorsal) do lado esquerdo.

As amostras de pele foram fixadas em solução de Bouin e Formol 10\% por 24 horas e, posteriormente, transferidas para concentrações crescentes de álcoois. Após a inclusão em parafina, as amostras de pele foram cortadas com aproximadamente $5 \mu \mathrm{m}$ de espessura e coradas pela técnica de hematoxilinaeosina (HE) (Luna, 1968) e Tricrômico de Masson (Michalany, 1980) para descrever a histologia geral da pele. Os cortes histológicos foram analisados pela microscopia de luz, selecionados e fotografados em fotomicroscópio Axiophot Zeiss.

\section{Método quantitativo}

Análises morfométricas foram realizadas na pele das três espécies para medir a espessura da derme da região dorsal de cada indivíduo antes do processo de curtimento.

Para cada individuo foram determinadas três medidas (mediana e laterais), com o auxílio de uma ocular de tambor 10x com filamento deslocável.

Antes da realização das medidas da derme, procedeu-se à calibração da ocular com uma lâmina especial (Carl Zeiss) provida de divisões de 0,01 $\mathrm{mm}$ $(10 \mathrm{~mm})$, visando transformar as unidades da ocular em micrômetros. Os valores medidos foram multiplicados pelo coeficiente micrométrico de cada objetiva e expressos em micrômetros $(\mu \mathrm{m})$. Todos os valores obtidos nas análises morfométricas tiveram sua média e desvios-padrão calculados.

Os dados de espessura da derme da pele na região dorsal antes do curtimento foram submetidos à análise de variância e as médias comparadas pelo teste Tukey (1 e 5\%).

\section{Processo de curtimento}

As peles utilizadas no curtimento foram retiradas dos peixes com auxílio de um alicate de ponta fina, a seguir colocadas individualmente em saco plástico, identificadas e congeladas para conservá-las até o momento da realização do curtimento.

Para o curtimento as peles foram descongeladas à temperatura ambiente e identificadas, com auxílio de miçangas coloridas de tamanhos diferentes, fixadas à pele por meio de uma linha.

As peles pesaram $2 \mathrm{~kg}$ e, a partir desse peso, foram calculadas as quantidades de água e produtos químicos adicionados em todas as etapas do curtimento. O processo de curtimento aplicado para as peles constou das seguintes etapas: remolho, caleiro, desencalagem, purga, píquel, curtimento, neutralização, recurtimento, tingimento, engraxe, secagem e acabamento. As etapas do processo de curtimento com suas finalidades estão listadas abaixo de acordo com Hoinacki (1989) e Hoinacki et al. (1994) e tendo a seguinte formulação:

Remolho: hidratação e lagavem das peles

$200 \%$ de água

$2 \%$ detensoativoSPO(Oleoquim)-10minutos-Esgotar $200 \%$ de água

$2 \%$ de tensoativo SPO

2\% de soda barrilha - 20 minutos - Esgotar

Caleiro: Intumescimento das peles, proporcionando a aberturas das fibras colágenas e liberação das escamas.

$200 \%$ de água

$3 \%$ de sulfeto de sódio

$2 \%$ de cal hidratada

$1 \%$ de tensoativo SPO - 2 horas

Esgotar

Observação : para o Piavuçu:

$2^{\circ}$ Caleiro:

$200 \%$ de água

$3 \%$ de sulfeto de sódio

$2 \%$ de cal hidratada

$1 \%$ de tensoativo SPO (Oleoquim) - 1h30

Esgotar

Lavar em água corrente

Desencalagem: eliminar o intumescimento alcalino e proporcionar afrouxamento da estrutura fibrilar.

$100 \%$ de água

$2,5 \%$ de sulfato de amônia

$0,4 \%$ de desencalante bascal (Basf)- 40 minutos

Purga: visa a limpeza da estrutura fibrosa com substâncias proteolíticas

$0,1 \%$ de Batan (Clariant)

$1 \%$ de tensoativo SPO - 30 minutos

Esgotar

Lavar 
Piquel: acidificar as fibras colágenas para reagirem com o curtente

$100 \%$ de água

$20 \%$ de cloreto de sódio (atingir $7,0^{\circ} \mathrm{Bé}$ ) - 10 minutos

$5 \%$ de ácido fórmico - $2 \mathrm{~h}$

Pernoitar no banho

Curtimento: proporcionar a estabilidade de todo o sistema colágeno, aumentando a temperatura de retração, diminuição da capacidade de intumescimento do colágeno e estabilização face às enzimas.

$10 \%$ de chromosal B (Basf) - 2 horas

Basificação:

$1,0 \%$ de bicarbonato de sódio - 1 hora

Esgotar

Neutralização ou desacidificação: eliminar o excesso de acidez do couro

$100 \%$ de água

$1,5 \%$ de bicarbonato de sódio - 1 hora

Esgotar

Lavar

Recurtimento e tingimento: proporcionar maior maciez ao couro e determinar a cor final do couro

$150 \%$ de água

$4 \%$ de relugan EXP (Basf)- 20 minutos

$6 \%$ de Tambit TA - 1 hora

$4 \%$ de Tambit TA

$2 \%$ de Basyntan LB2 (Basf)

1,5\% de Resina acrílica - DC 747

$2,5 \%$ de relugan EXP (Basf)

$3 \%$ de corante NGB (Basf)- 1 hora

Rodar fulão com água aquecida a $60^{\circ} \mathrm{C}-10$ minutos

Esgotar

Lavar com água a $60^{\circ} \mathrm{C}$

Engraxe: proporcionar a maciez e elasticidade do couro pela ação dos óleos envolvendo as fibras colágenas, evitando a aglutinação dessas fibras.

$150 \%$ de água a $60^{\circ} \mathrm{C}$

$4 \%$ de Lipodermilicker LA NAE (Basf)

$4 \%$ de Lipodermilicker LA-2 (Basf)

$1 \%$ de Lipodermilicker LBCT (Basf) - 40 minutos

1,5\% de ácido fórmico - 2x 15 minutos - Esgotar Lavar

Secagem à temperatura ambiente : reduzir o teor de água

Amaciamento: proporcionar o deslizamento das fibras colágenas umas sobre as outras.

Prensagem: deixar o couro liso evitando-se dobras.

Para a pele de piavuçu houve necessidade de realizar um segundo caleiro, pois a pele não intumesceu o suficiente, não apresentando as características de estarem translúcidas e intumescidas.

\section{Análise físico-mecânica}

Foram utilizados 10 couros obtidos a partir das peles curtidas de cada espécie de peixe. As amostras analisadas foram retiradas no sentido longitudinal ao comprimento do peixe, próximo à região dorsal do peixe e identificadas.

A seguir, as amostras foram levadas ao laboratório, em ambiente climatizado a $23 \pm 2^{\circ} \mathrm{C}$ e umidade relativa do ar de 50 $\pm 2 \%$, conforme NBR 10455 (1988), por um período de 24 horas Os testes foram realizados com dinamômetro EMIC, com velocidade de afastamento entre cargas de $100 \pm 20 \mathrm{~mm} / \mathrm{mm}$. Antes de realizar os testes físico-mecânicos, determinaram-se as medidas de espessura das amostras, de acordo com NBR 11062 (1997).

\section{Resultados e Discussão}

\section{Análise morfológica}

A pele das três espécies analisadas (Leporinus macrocephalus, Brycon hilarii e Mylossoma sp.) é formada por três camadas distintas: epiderme, derme e hipoderme. Todavia, as análises relatadas a seguir restringem-se à camada dérmica.

Leporinus macrocephalus - A pele do piavuçu (L. Macrocephalus) é constituída por uma epiderme e uma derme subjacente. Abaixo da membrana basal observa-se uma derme frouxa com fibras colágenas finas; melanóforos descontínuos e vasos sangüíneos.

A derme compacta é formada por tecido conjuntivo denso modelado (Figura 1). Entre as fibras colágenas espessas podem-se notar alguns fibroblastos. Na derme compacta, também foram observados, em alguns locais, grupos de células adiposas grandes e em outros, uma derme compacta com fibras colágenas espessas, onduladas e paralelas à superfície da epiderme. Souza \& Leme dos Santos (1995b) e Dourado et al. (1997) observaram esse mesmo padrão quanto à disposição das fibras colágenas, respectivamente, para a pele do curimbatá (Prochilodus lineatus) e tucunaré (Cichla ocellaris). Entre a derme compacta e a camada subjacente, ou seja, a hipoderme, verificaram-se melanóforos descontínuos.

R. Bras. Zootec., v.32, n.6, p.1551-1559, 2003 (Supl. 1) 
Brycon hilarii - A derme da piraputanga (Brycon hilarii) apresenta-se dividida em duas camadas: uma superficial ou frouxa, subjacente à epiderme, composta por fibras colágenas finas, com melanóforos e vasos sangüíneos e no estrato mais profundo (derme compacta) as fibras colágenas mostram-se espessas e paralelas em relação à epiderme (Figura 2). Em alguns pontos da derme, podem ser observadas fibras colágenas no sentido transversal. De acordo com Junqueira et al. (1983a), esse padrão descrito para a derme profunda é muito comum para as peles dos peixes.

Mylossoma sp - No pacu prata (Mylossoma sp.) o padrão tegumentar segue aquele descrito para a espécie piraputanga. No tecido conjuntivo frouxo subjacente à epiderme, as fibras colágenas são finas e vistas entre os vasos sangüíneos e melanóforos. Alguns linfócitos podem ser notados no tecido conjuntivo frouxo abaixo da epiderme. A derme compacta possui fibras colágenas espessas, paralelas e
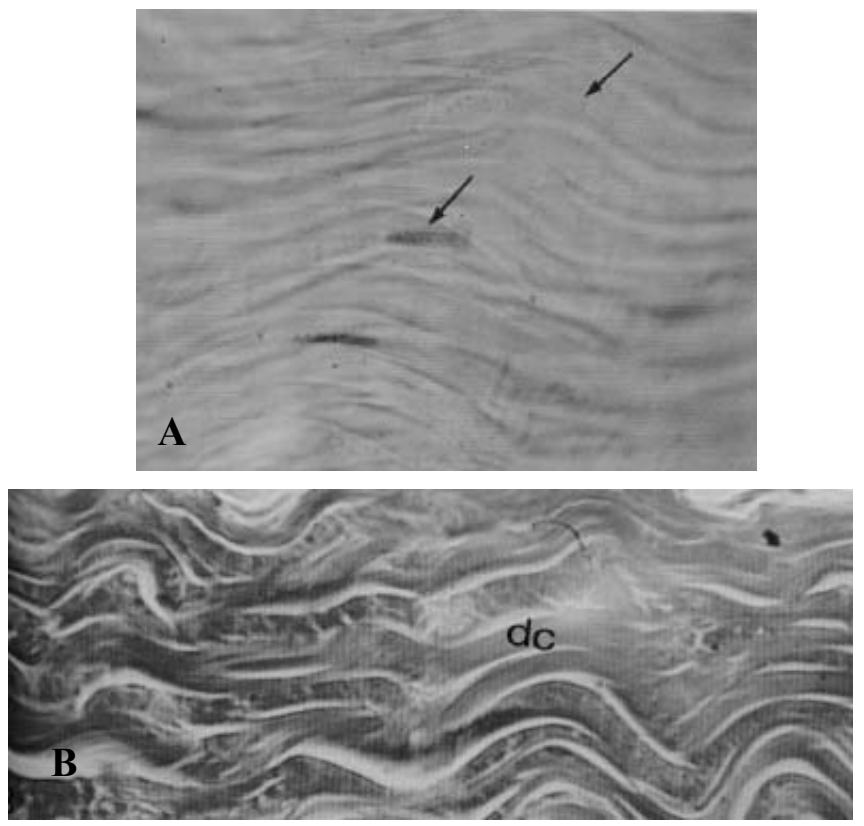

Figura 1 - Fotomicrografia da derme piavuçu (L. Macrocephalus) mostrando o arranjo das fibras colágenas da derme compacta (seta ou dc), na região dorsal do peixe. (A) aumento $=1000 \times$ e coloração $=\mathrm{HE}$. (B) aumento=1000X e coloração TM.

Figure 1 - Photomicrography of piavuçu (L. Macrocephalus) dermis showing the setting of collagen fibers of compact dermis (arrow and dc) in the fish dorsal region. $(A)$ increase $=1000 X$ and staining $=H E ;(B)$ increase $=1000 \mathrm{X}$ and staining $=T M$. imbricadas semelhantes ao arranjo de telhas de um telhado. Entre a derme compacta e a hipoderme observam-se melanóforos descontínuos, ou seja, sem formar camada contínua destas células pigmentares (Figura 3). O mesmo foi observado por Souza \& Leme dos Santos (1995a), para a pele de tilápia do Nilo, e Lorentz et al. (1999), para a pele do piaussu.

\section{Análise morfométrica}

A análise morfométrica da espessura da derme da pele das três espécies de peixes antes do curtimento. Constatou-se que a média da espessura da derme dorsal do piavuçu $(21,56 \mathrm{~mm})$ é maior que a média da espessura da derme dorsal do pacu $(20,88 \mathrm{~mm})$. A espessura na piraputanga $(18,04 \mathrm{~mm})$ é inferior à espessura da derme das duas espécies analisadas (Figura 4).

\section{Análise físico-mecânica}

Tendo-se em vista a complexidade do processo de transformação da pele em couro para sua utilização em
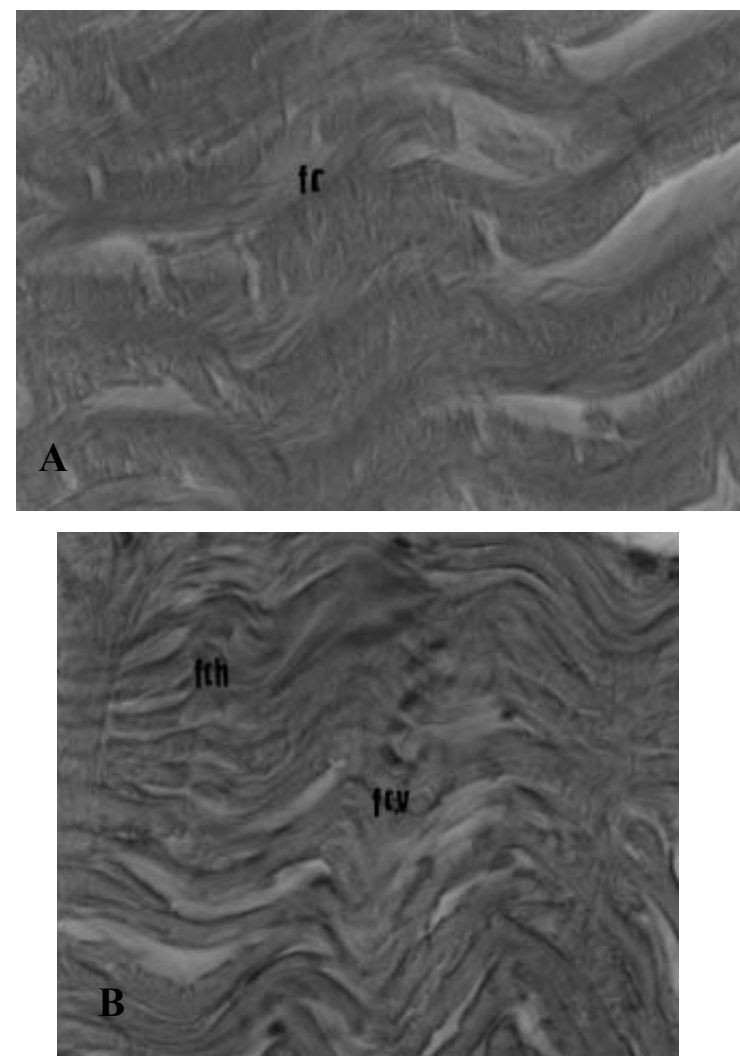

Figura 2 - Fotomicrografia da derme da piraputanga (Brycon hilarii) mostrando as fibras colágenas espessas (fc) e paralelas à superfície (fch) e transversais (fcv). (A e B) Aumento $=1000 \mathrm{Xe}$ Coloração $=\mathrm{HE}$

Figure 2 - Photomicrography of piraputanga (Brycon hilarii) dermis showing the thick collagen fibers $(f c)$ parallel to the surface (fch) and transversal (fcv). ( $A$ and $B$ ) Increase $=1000 \mathrm{X}$ and Staining $=\mathrm{HE}$. 
artigos, tais como vestuários, calçados, cintos, bolsas entre outros, há a necessidade da determinação da sua resistência como couro. Todavia, não existem parâmetros específicos para couros de peixes.

Os testes de resistência à tração e ao alongamento realizados nos couros das três espécies de peixes constam na Tabela 1 e a determinação da força de rasgamento progressivo, na Tabela 2 .

O couro do piavuçu $\left(18,50 \mathrm{~N} / \mathrm{mm}^{2}\right)$ e da piraputanga $\left(16,88 \mathrm{~N} / \mathrm{mm}^{2}\right)$ apresentaram maior resistência à tração quando comparado com couro do pacu $\left(11,83 \mathrm{~N} / \mathrm{mm}^{2}\right)$. Os resultados obtidos nesses dois couros (piavuçu e piraputanga) foram próximos aos relatados por Pederzolli et al. (1995) para o couro de Cyprinus carpio $\left(15,47 \mathrm{~N} / \mathrm{mm}^{2}\right)$. Todavia, os valores foram inferiores ao obtido por Almeida (1998) para couro de tilápia com $0,41 \mathrm{~mm}$ de espessura, de $22,16 \mathrm{~N} / \mathrm{mm}^{2}$.

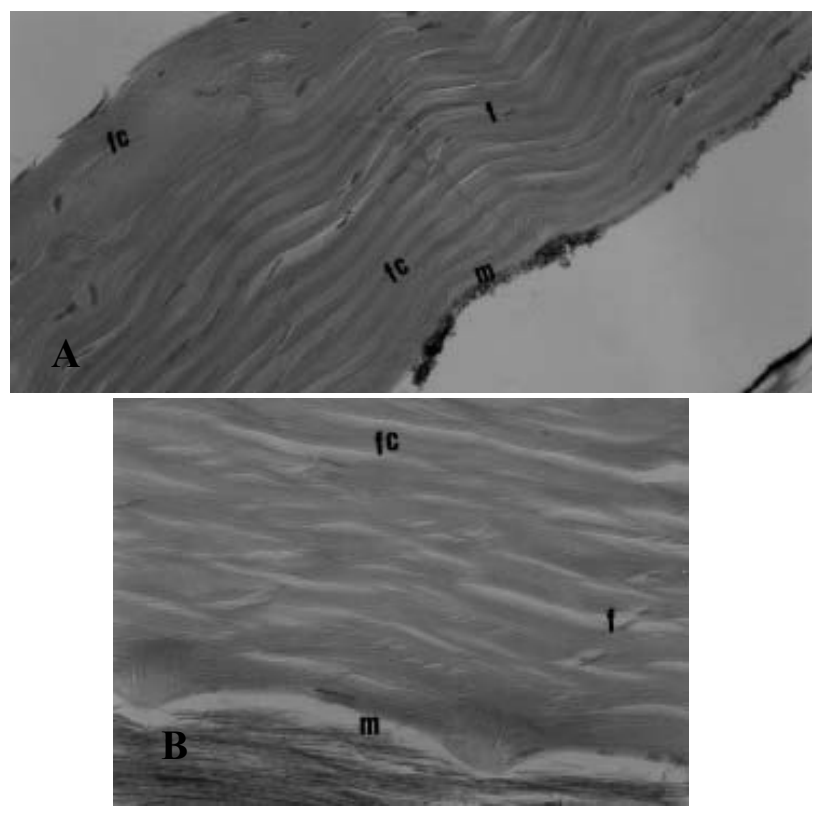

Figura 3 - Fotomicrografia da derme do pacu prata (Mylossoma sp) mostrando: (A) fibras colágenas de diferentes espessuras (fc); fibroblastos (f); fina camada de melanóforos entre a derme e hipoderme $(\mathrm{m})$. Aumento $=400 \mathrm{X}$ e Coloração $=$ HE. (B) delgadas fibras colágenas paralelas à superfície (fc); fibroblastos (f). Aumento $=400 \mathrm{X}$ e Coloração = HE.

Figure 3 - Photomicrography of pacu prata (Mylossoma sp) dermis showing: (A) collagen fibers of different thickness (fc); fibroblasts (f); a thin layer of melanofores between the compact dermis and hypodermis $(\mathrm{m})$. Increase $=400 X$ and Staining $=H E$. $(B)$ thin collagen fibers parallel to the surface (fc); fibroblasts $(f)$. Increase $=400 X$ and Staining $=H E$.
Quanto à porcentagem de alongamento ou elongação até a ruptura, também não houve diferença significativa $(\mathrm{P}<0,05)$ para piavuçu $(52,83 \%)$ e piraputanga $(60,45 \%)$, sendo estes significativamente superiores ao de pacu $(33,83 \%)$. Estes valores foram inferiores ao apresentado por Almeida (1998) para a tilápia (79\%), no entanto tais resultados obtidos para piavuçu e piraputanga foram semelhantes aos relatados por Pederzolli et al. (1995) para Monacanthus hispidus (máx. 54\%) e Cyprinus carpio (máx. 60\%).

A carga de força em $\mathrm{N}$ resultante do teste de resistência à tração e alongamento foi significativamente diferente $(\mathrm{P}<0,01)$. Os valores obtidos para o couro de pacu $(43,75 \mathrm{~N})$ e piraputanga $(47,51 \mathrm{~N})$ não diferiram entre si, mas foram inferiores $(\mathrm{P}<0,01)$ ao do piavuçu $(115,65 \mathrm{~N})$. Os resultados da carga de força aplicada nesse teste para os couros de pacu e piraputanga foram inferiores ao relatado por Almeida (1998) para o couro de Oreochromis niloticus (92,18 N), enquanto o resultado do piavuçu foi superior ao relatado por esse mesmo autor.

Apesar da menor espessura do couro de piraputanga $(0,29 \mathrm{~mm})$ comparativamente aos demais couros analisados, os testes de resistência à tração $\left(16,88 \mathrm{~N} / \mathrm{mm}^{2}\right)$ e valor de elongação $(60,45 \%)$ foram superiores aos resultados obtidos no couro de

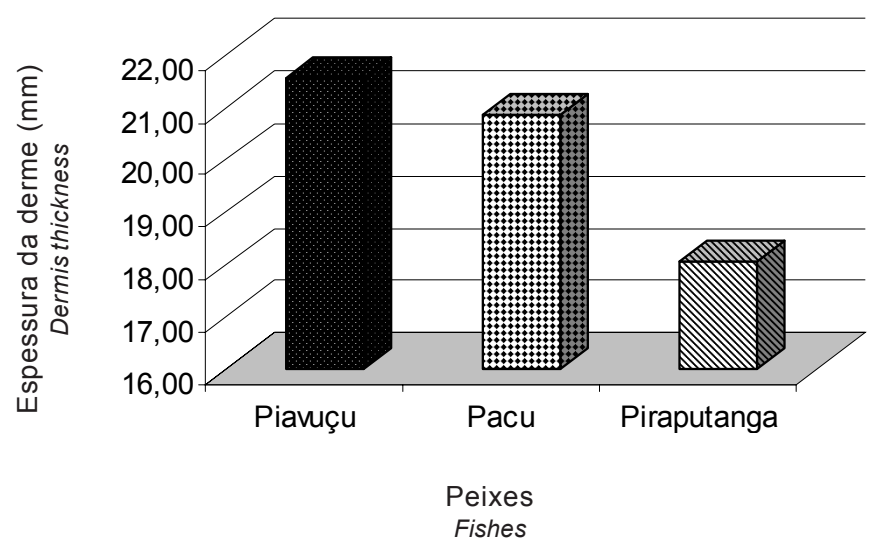

Figura 4 - Valores médios da espessura da derme da região dorsal dos peixes: piavuçu (L. Macrocephalus), pacu prata (Mylossoma $\mathrm{sp})$ e piraputanga (Brycon hilarii).

Figura 4 - Mean values of dermis thickness of piavuçu ( $L$. Macrocephalus), pacu prata (Mylossoma $s p$ ) and piraputanga (Brycon hilarii) dorsal regions. 


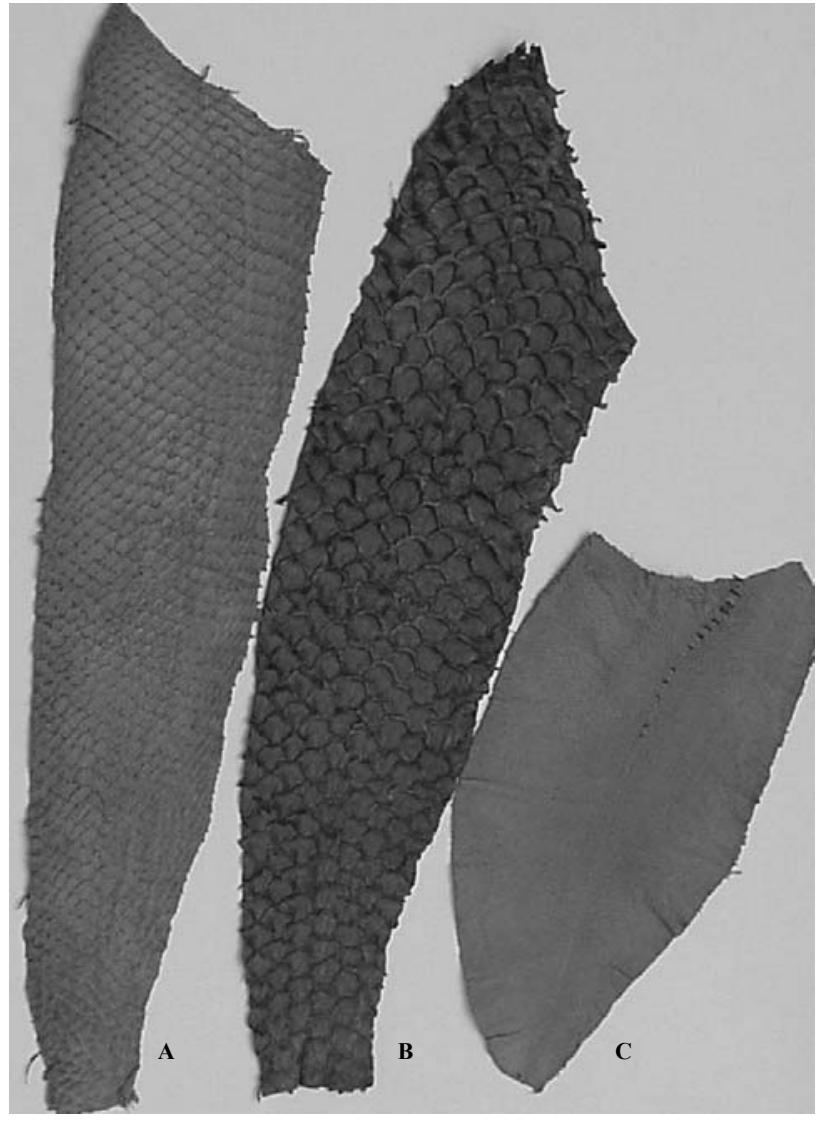

Figura 5 - Pele da piraputanga $(A)$, piavuçu $(B)$ e pacu prata (C) após o curtimento.

Figura 5 - Skin of piraputanga $(A)$, piavuçu (B) and pacu prata (C) after hardening. pacu. Isso pode ser explicado pela disposição das fibras colágenas na pele de piraputanga, que, além de estarem distribuídas paralelamente à epiderme, também se encontram dispostas no sentido transversal à epiderme, formando um entrelaçamento dos feixes de fibras colágenas, proporcionando maior resistência ao couro. Todavia, os resultados de resistência à tração e valor de elongação da piraputanga não diferiram do piavuçu, podendo ser justificados pela elevada espessura do couro nessa espécie de peixe $(0,61 \mathrm{~mm})$. A espessura desse couro foi superior à espessura dos demais couros (Tabela 1).

De acordo com Junqueira et al. (1983b), o arranjo estrutural das fibras colágenas da derme compacta, bem como a espessura desse estrato, permite que a pele possua grande resistência às diferentes forças de tração. Por essa razão, a pele de algumas espécies de peixes pode ser utilizada comercialmente na confecção de artefatos de couro.

Os resultados de rasgamento progressivo para os couros de piraputanga e piavuçu foram significativamente inferiores (Tabela 1$)$ ao de pacu $(36,51 \mathrm{~N} / \mathrm{mm})$. Não houve diferença significativa entre o couro de piraputanga $(8,45 \mathrm{~N} / \mathrm{mm})$ e piavuçu $(8,16 \mathrm{~N} / \mathrm{mm})$. $\mathrm{O}$ valor obtido para pacu foi próximo ao de Monacanthus hispidus $(34,2 \mathrm{~N} / \mathrm{mm})$ e Cyprinus carpio $(32,7 \mathrm{~N} / \mathrm{mm})$ (Pederzolli et al., 1995).

As fibras colágenas da derme do couro de pacu, observadas na análise histológica, estavam imbricadas,

Tabela 1 - Médias de espessura, resistência à tração e alongamento de couro de piavuçu, piraputanga e pacu Table 1 - Means of thickness, resistance to traction and elongation of leather of piavuçu, pacu and piraputanga

\begin{tabular}{|c|c|c|c|c|}
\hline \multirow[t]{2}{*}{$\begin{array}{l}\text { Couro } \\
\text { Leather }\end{array}$} & \multirow[t]{2}{*}{$\begin{array}{l}\text { Espessura }(\mathrm{mm}) \\
\text { Thickness }\end{array}$} & \multicolumn{3}{|c|}{$\begin{array}{l}\text { Resistência à tração e alongamento } \\
\text { Resistance to traction and elongation }\end{array}$} \\
\hline & & $\begin{array}{l}\text { Carga }(\mathrm{N}) \\
\quad \text { Load }\end{array}$ & $\begin{array}{c}\text { Tensão à tração }\left(\mathrm{N} / \mathrm{mm}^{2}\right) \\
\text { Tension to traction }\end{array}$ & $\begin{array}{c}\text { Valor de elongação (\%) } \\
\text { Value of elongation }\end{array}$ \\
\hline Piavuçu & $0,61 \mathrm{a}^{(1)}$ & $115,65 a$ & $18,50 \mathrm{a}$ & $52,83 a$ \\
\hline Piraputanga & $0,29 \mathrm{c}$ & $47,51 b$ & $16,88 \mathrm{a}$ & $60,45 a$ \\
\hline $\mathrm{Pacu}$ & $0,37 b$ & $43,75 b$ & $11,83 b$ & $33,83 b$ \\
\hline Teste F & $78,01 * *$ & $50,47 * *$ & $12,54 * *$ & $30,05^{*}$ \\
\hline$F$ test & & & & \\
\hline $\mathrm{CV}(\%)$ & 13,97 & 26,12 & 19,75 & 16,12 \\
\hline
\end{tabular}

${ }^{1}$ Em cada coluna, valores seguidos de mesma letra não diferem pelo teste Tukey a $5 \%$ de probabilidade.

${ }_{1}$ In each column, values followed by the same letter do not differ by Tukey test at a $5 \%$ probability. 
Tabela 2 - Médias de espessura e força de rasgamento progressivo do couro de piavuçu, piraputanga e pacu Table 2 - Mean thickness and strength of leather progressive tearing of piavuçu, piraputanga and pacu

\begin{tabular}{|c|c|c|c|c|}
\hline \multirow[t]{2}{*}{$\begin{array}{l}\text { Couro } \\
\text { Leather }\end{array}$} & \multirow[t]{2}{*}{$\begin{array}{l}\text { Espessura }(\mathrm{mm}) \\
\text { Thickness }\end{array}$} & \multicolumn{3}{|c|}{$\begin{array}{l}\text { Força de rasgamento progressivo } \\
\text { Strength of progressive tearing }\end{array}$} \\
\hline & & $\begin{array}{l}\text { Carga }(\mathrm{N}) \\
\quad \text { Load }\end{array}$ & $\begin{array}{l}\text { Rasgo }(\mathrm{N} / \mathrm{mm}) \\
\text { Tearing }\end{array}$ & $\begin{array}{l}\text { Força máxi ma }(\mathrm{N}) \\
\text { Maximun strength }\end{array}$ \\
\hline Piavuçu & $0,74 \mathrm{a}^{(1)}$ & $6,18 \mathrm{~b}$ & $8,45 \mathrm{~b}$ & $17,05 \mathrm{~b}$ \\
\hline Piraputanga & $0,30 \mathrm{c}$ & $2,49 \mathrm{c}$ & $8,16 \mathrm{~b}$ & $6,72 \mathrm{c}$ \\
\hline Pacu & $0,51 b$ & $18,73 \mathrm{a}$ & $36,51 \mathrm{a}$ & $44,30 \mathrm{a}$ \\
\hline Teste F & $77,62 * *$ & $171,19 * *$ & $277,88^{* *}$ & $186,26^{* *}$ \\
\hline$F$ test & & & & \\
\hline CV $(\%)$ & 14,42 & 21,36 & 16,55 & 18,81 \\
\hline
\end{tabular}

${ }^{1}$ Em cada coluna, valores seguidos de mesma letra não diferem pelo teste Tukey a 5\% de probabilidade.

1 In each column, values followed by the same letter do not differ by Tukey test at a $5 \%$ probability.

dificultando o seu rasgamento, portanto, a carga de força $(18,73 \mathrm{~N})$ e a força máxima $(44,30 \mathrm{~N})$ utilizada no teste para obtenção do rasgamento progressivo $(36,51 \mathrm{~N} / \mathrm{mm})$ foram significativamente $(\mathrm{p}<0,01) \mathrm{su}-$ periores aos resultados observados nos couros de piavuçu e piraputanga (Tabela 2).

Quanto à utilização destes tipos de couro para a indústria do vestuário e de calçados, pode-se observar que o couro de piraputanga, piavuçu e pacu é adequado para utilização no vestuário, já que, de acordo com Niveles de calidad aceptables em la indústria del cuero (1976), citado por Hoinacki (1989), os valores orientados para vestuário curtido ao cromo, o couro deve apresentar resistência à tração de, no mínimo, $9,80 \mathrm{~N} / \mathrm{mm}^{2}$. Por outro lado, o couro de piraputanga e pacu não deve ser indicado para utilização para cabedal de calçados, pois, segundo a Escola de Curtimento SENAI, exige-se no mínimo uma resistência à tração de $17,65 \mathrm{~N} / \mathrm{mm}^{2}$. Todavia, o couro piavuçu $\left(18,50 \mathrm{~N} / \mathrm{mm}^{2}\right)$ está dentro do valor exigido para cabedal de calçados.

Quanto aos critérios relacionados com a elongação, os couros de piraputanga e piavuçu podem ser utilizados para confecção de vestuários, pois, de acordo com Vademécum para el técnico em curtición BASF, citado por Hoinacki (1989), o valor orientado para utilização do couro para vestuário curtido ao cromo é de $60 \%$ de elongação até a ruptura.

Além desses aspectos abordados referentes às análises físico-mecânicas e histológica da derme, torna-se importante mencionar o aspecto visual da pele após o seu processamento (curtimento) (Figura 5).
Segundo Almeida (1998), o desenho da flor da pele é caracterizado pelas lamélulas de proteção e inserção das escamas, formando mosaicos inimitáveis na pele de peixes de escamas. O desenho é uma característica própria de cada espécie após o curtimento, constituindo uma definição de cada tipo de pele, em função da espécie.

Esse desenho da flor pode ser observado no couro proveniente das três espécies de peixes estudados, estando em função ao tamanho do local onde as escamas estavam inseridas na pele e o comprimento dessas lamélulas (Figura 5). Observando as três espécies de peixes, verifica-se que a pele do piavuçu proporciona um couro com uma característica de desenho da flor mais definida e de difícil imitação, devido ao maior tamanho das lámelulas de proteção e inserção das escamas desse peixe, enquanto no couro da piraputanga e pacu esse desenho proporcionado pelas lamélulas de inserção das escamas é muito discreto.

\section{Conclusões}

As peles das espécies de peixe apresentaram diferenças histológicas quanto à disposição e distribuição das fibras colágenas da derme.

As peles das três espécies analisadas podem ser transformadas em couro para sua aplicação em diversos artefatos, inclusive para utilização na confecção de vestuários. Todavia, o couro de piavuçu poderia ser usado em calçados, enquanto os demais couros somente poderiam ser utilizados na forma de 
apliques ou com revestimento apropriado (couro bovino). O couro de piavuçu apresenta um desenho da flor mais definido e de difícil imitação.

\section{Agradecimento}

À CAPES, pelo auxílio da bolsa PICDt; à empresa Projeto Pacu, por ter gentilmente cedido os peixes para a realização deste trabalho; ao biológo Helder A. Souza, que muito contribuiu na realização do mesmo; ao Sr. Attila Ko Freitag do Curtume MS Leather, pelo apoio na execução do curtimento das peles; e aos técnicos da Leather Solutions e BASF, pelo apoio na realização das análises de resistência do couro.

\section{Literatura Citada}

ABRACOA,1995. Disponível em: http://www.setorpesqueiro.com.br acesso: 15 Set.2001.

ADEODATO, S. Peles exóticas e ecológicas. Globo Ciência, v.51, p.56-60, 1995.

ALMEIDA, R.R. A pele de peixe tem resistência e flexibilidade? Revista do Couro, v.127, p.49-53, 1998.

CONTRERAS-GUZMÁN, E.S. Bioquímica de pescados e derivados. Jaboticabal: FUNEP, 1994. 409p.

DOURADO, D.M.; SOUZA, M.L.R.; LEME DOS SANTOS, H.S. et al. Histological analysis of tucunaré (Cichla ocellaris), captured from Piquiri River Basin, Alto Pantanal (MS), Brazil. In: THE ANNUAL INTERNATIONAL CONFERENCE \& EXPOSITION OF THE WORLD AQUACUlTURE SOCIETY, 1997, Seatle, Abstracts... Seatle: World Aquaculture Society, 1997. p.128.

HOINACKI, E. Peles e couro: origens, defeitos e industrialização. 2.ed. Porto Alegre: CFP de Artes Gráficas "Henrique d'Ávila Bertaso", 1989. 319p.

HOINACKI, E.; MOREIRA, M.V.; KIEFER, C.G. Manual básico de processamento do couro. Porto Alegre: SENAI, Estância Velha, Centro Tecnológico do Couro, 1994. 402p.

JUNQUEIRA, L.C.U.; JOAZEIRO, P.P.; MONTES, G.S. et al. The collagen fiber architecture of brasilian naked skin. Brazilian Journal Medicinal Biologycal Research, v.16, p.313-316, 1983a.

JUNQUEIRA, L.C.U.; JOAZEIRO, P.P.; MONTES, G.S. et al. É possível o aproveitamento industrial da pele dos peixes de couro? Tecnicouro, v.5, n.5, p.4-6, 1983 b.
LORENTZ, D.N.; DOURADO, D.M.; SOUZA, C.C. et al. Estudo histológico, histoquímico e morfométrico da pele do piaussu (Leporinus macrocephalus), capturado nos rios Miranda e Aquidauana. Ensaios e Ciências, v.3, n.2, p.85-112, 1999.

LUNA, L.G. Manual of histologic staiting methods of Armed Forces Institute of Phatology. 3.ed. New York: Mc GrawHill Book Company, 1968. 257p.

MICHALANY, J. Técnica histológica em anatomia patológica. 1.ed. São Paulo: Ed. Pedagógica e Universitária, 1980. $277 \mathrm{p}$.

NBR 10455 - NORMA BRASILEIRA REGISTRADA, SISTEMA NACIONAL DE METROLOGIA, NORMATIZAÇÃO E QUALIDADE INDUSTRIAL - Climatização de materiais usados na fabricação de calçados e correlatos. Procedimento. 1988. p.2-3.

NBR 11062 - NORMA BRASILEIRA REGISTRADA, SISTEMA NACIONAL DE METROLOGIA, NORMATIZAÇÃO E QUALIDADE INDUSTRIAL - Determinação da espessura. Procedimento. 1997.

PEDERZOLLI, A. R.; SCHEIBE, E.; STREIT, K.F. et al. Study of the economical viability of processing of fish skins. In: CONGRESS OF THE INTERNATIONAL UNION LEATHER TECHNOLOGISTS AND CHEMISTS SOCIETIES, 23., 1995, Friedrichshafen. Proceedings... Friedrichshafen, 1995. (Artigo 40)

SACCO, D. Curtimento transforma pele de peixe em objetos da moda. Revista Unesp Rural, v.12, p.24-26, 1998.

SOUZA, M.L.R.; LEME DOS SANTOS, H.S. Análise microscópica comparada da pele da tilápia (Oreochromis niloticus), da carpa espelho (Cyprinus carpio specularis) e carpa comum (Cyprinus carpio). In: SEMANA SOBRE HISTOLOGIA DE PEIXES, FCAVJ-UNESP, 2., 1995, Jaboticabal. Resumos e palestras... Jaboticabal: FUNEP, 1995a, p.100-101.

SOUZA, M.L.R.; LEME DOS SANTOS, H.S. Análise microscópica comparada da pele do pacu (Piaractus mesopotamicus), do tambacu (macho pacu x fêmea tambaqui) e do curimbatá (Prochilodus lineatus). In: SEMANA SOBRE HISTOLOGIA DE PEIXES, FCAVJ-UNESP, 2., 1995, Jaboticabal. Resumos e palestras... Jaboticabal: FUNEP, 1995b. p.105-106.

Recebido em: 13/05/02 Aceito em: 07/10/02 